Sharif University of Technology
Scientia Iranica
SCIENTIA $\quad \begin{gathered}\text { Transactions D: Computer Science ES Engineering and Electrical Engineering } \\ \text { http://scientiairanica.sharif.edu }\end{gathered}$

\title{
Educational robot for principles of electrical engineering
}

\author{
M. Rahnavard, S.M.H. Alavi, S. Khorasani*, M. Vakilian, and M. Fardmanesh \\ School of Electrical Engineering, Sharif University of Technology, Tehran, Iran.
}

Received 30 January 2016; received in revised form 28 September 2016; accepted 7 November 2016

\section{KEYWORDS}

Electrical engineering education;

Educational robot;

Principles of electrical

engineering;

Laboratory-based

education.

\begin{abstract}
An educational robot is described, which is designed and constructed for use in the laboratory of Principles of Electrical Engineering. The discrete nature of design, conforming to a simple block strategy, allows for fairly easy introduction of basic and fundamental concepts of Electrical Engineering to the freshman students, including control, actuation, wireless signal transmission, and analogue/digital conversions. Each block or module represents one or few engineering ideas and helps the students to understand the interplay and connection between system divisions, and enhance their creative thinking. Circuits are designed and implemented such that both structure and functionality of each module are clearly presented. Inputs and outputs as well as major signals can be traced and measured through several test points.
\end{abstract}

(C) 2018 Sharif University of Technology. All rights reserved.

\section{Introduction}

Among the modern sciences, Electrical Engineering (EE) has deep roots in basic sciences, especially mathematics and physics. In 1882, the first course on EE was offered as an optional course in the Department of Physics at Massachusetts Institute of Technology. From 1903 through the early days of World War II, EE was based on the education of engineering skills, facts, and methods as demanded by the industry $[1,2]$. However, major breakthroughs such as Radio and RADAR were accomplished by the Electrical Engineers educated in these years. It was not until after this period of time that a modern educational system was founded on strengthening mental skills, rather than solely educating skills and practices.

In the year 1978, the Institute of Electrical and

*. Corresponding author.

E-mail address: khorasani@sina.sharif.edu (S. Khorasani)

doi: $10.24200 /$ sci. 2017.4369
Electronics Engineers (IEEE) set up a committee together with representatives from industries, which devised a curriculum for EE; this curriculum was intended to extend engineering proficiencies rather than methods needed by industries. In 1994, the US National Science Foundation (NSF) ordered revisions in science and engineering curricula to improve creativity in the educational system of engineering disciplines. Since then, many efforts have been made to identify the weaknesses and strengths of the educational methodology in EE [38]. Of particular interest has been better en gagement of female students in the area of EE [9]. These objectives can be met only if the students have a deeper and better engineering sense. Furthermore, there has been a need for sustained improvement in the quality of graduated engineers [10] along with the rapid advancement in science and technology, which was delayed for years owing to the old-fashioned and classic systems of education in use. One of the major paths towards meeting the objectives of a modern educational system is to present fundamental engineering concepts to freshman students in the first semester. In this system, the students are 
given an opportunity to obtain a wide view of EE along with deep understanding of fundamental concepts, thus getting involved in obtaining the necessary knowledge and skills of engineering.

In the traditional curriculum of Iran, undergraduate students of $\mathrm{EE}$ in the first year are only exposed to advanced levels of mathematics and physics; hence, before the end of their first year, they have no idea about EE. Considering the importance of time in absorbing the concepts, an introduction to the fundamentals of EE in the first year should have a critical role in improving the learning efficiency of students. Hence, the School of EE at Sharif University of Technology (SUT) has devised a fully amended curriculum, in which the freshman students need to pass a 4-unit course entitled Principles of Electrical Engineering. This novel method of combined courseplus-laboratory offering has been in practice constantly since then, and extended to many other undergraduate courses. Duke University was also among the first institutions to revise their whole curriculum of EE, and they designed a laboratory based course entitled Fundamentals of Electrical and Computer Engineering for freshman students [11].

In this course, the students are exposed and introduced to various disciplines of EE, while in laboratory they experiment with EE from discrete elements all the way to an elaborate example system: a remote-control multifunctional educational robot. The reason behind this choice is clear; the robots could elegantly present a combination of many engineering disciplines in a single attractive system. The educational value of robots for this purpose was soon recognized [12-14], which would offer versatile learning platforms to the students.

Most existing educational robot designs are based on Integrated Circuits (ICs), Field Programmable Gate Arrays (FPGAs), or embedded processing units [1419]. Expectedly, the use of robots in education of students at the university level has been on rise, drawing significant attraction among academia in the recent years [20-48]. However, a survey reported in [20] also justified the conclusion that among numerous educational robot designs, no one would be applicable to the education of electronic circuits. The reason is that the progress has been toward high-integration and software-control, instead of system breakdown and modular design. Nao [49] is a famous example of such an advanced and versatile robot, to which students have only external software access.

Therefore, the main advantage of the implemented design in this paper is the use of components with a low level of integration, which allows the freshman student to deeply understand the typical operation and connection of circuits and components in a real complex system. Therefore, the motivation is to fully understand the operation of basic subsystems and enhance the creative thinking using only discrete or low integration components. Access to important signals and the possibility of measurements via standard equipment have been considered, too. These particular features of the implemented robot would be advantageous in system-level breakdown compared to other similar educational robots. By using this robot, students observe how a complicated system can be made like a jigsaw puzzle, every piece of which is obtained from circuits made out of basic discrete electrical, electronic elements and integrated circuits. This design aligns very well with the necessity of an accelerated educational program [50] while developing creative engineering skills.

\section{Structure of principles of electrical engineering}

The 4-units course of Principles of Electrical Engineering consists of 3 units of theory in the class and 1 unit in the laboratory offered to the first-semester students. The major theme of this course is to introduce students to the fundamental and basic concepts of EE from an application and engineering point-of-view so that at the end of the semester, the students would be able to sense and analyze simple electrical and electronic circuitbased systems. The course comprises all fields of EE and for every topic, there exists a suitable experiment. All circuits are modular and packed in transparent containers to display the actual elements.

It should be pointed out that despite the simplicity of the course syllabus, all of the instructed material is used in the upcoming courses and the students learn the subjects in such a way that no major repetition of content would be needed later. In addition, the students are requested to carry out exercises in electrical and electronic circuits with SPICE simulation software where they can compare the practical and theoretical/simulation results.

Students get acquainted with various fields of EE through illustration of concepts and attractive applications in Communications, Power, Microelectronics, Biomedical, and Control Engineering at an understandable level for a freshman student. In the meantime, selected professors from those fields are invited to deliver lectures within their own fields of expertise in order to provide maximum benefit for the students. While encouraging students to participate in the lectures, it is anticipated that future graduates of EE will have a much deeper and wider understanding and knowledge with respect to the applications and relation between theory and practice. The last laboratory session is devoted to an experiment, which is a kind of collective teaching of various introduced aspects of EE in the course based on a remote-control modular robot, as described next. 


\section{Educational robot}

The implemented educational robot as shown in Figure 1 consists of two parts: The first part is the remote-control unit by which the user can control the robot car; the second part is the robot itself, which receives and executes the commands. In Figure 2, the block diagram of the control unit and in Figure 3, the structure of the robot are illustrated. As shown in Figure 3, the control unit sends several commands to the robot. These include 2 analog commands, namely, velocity and direction, and 6 digital commands in the form of 6 on-off switches, which are shown on the left. Velocity and direction commands are converted to digital using two 4-bit Analogue-to-Digital (A/D) converters. Digital data is displayed on a basic LED display. Then, the digital data is converted from the parallel to serial arrangement. The serial data is PulseWidth-Modulation (PWM) modulated, and at last,

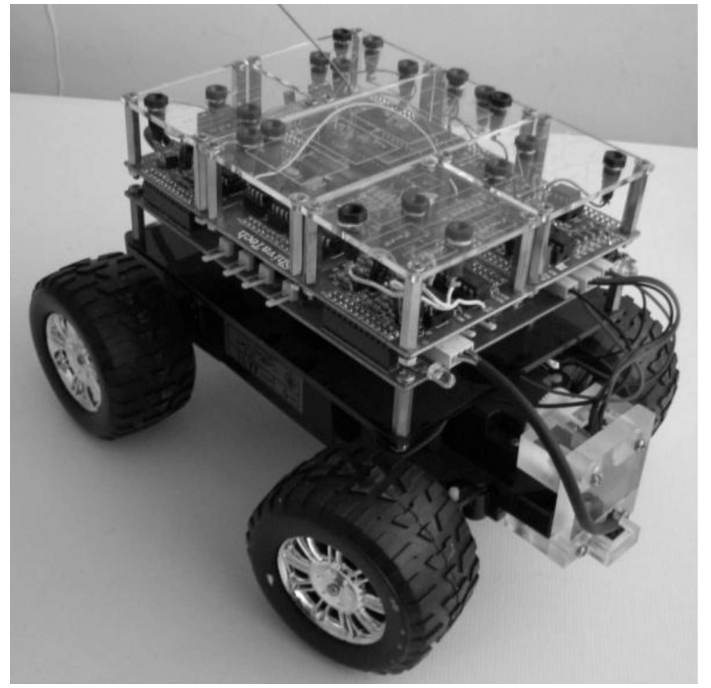

Figure 1. Photo of the educational robot. the PWM signal is Amplitude-Modulated (AM) in the $27 \mathrm{MHz}$ band for transmission to the robot.

In the robot, the data on air is received as serial data by a super-heterodyne radio. Clock and data are separated from the serial data, and the data is restored to the parallel form and displayed on the local LightEmitting-Diode (LED) display as in the control unit. Digital commands corresponding to velocity and direction are sent as 4-bit signals to 2 Digital-to-Analogue (D/A) converters. The analog output is amplified and level-shifted to obtain the necessary command voltages. Two voltages corresponding to velocity and direction are applied to 2 motor driver circuits to control the drive and steering motors. The prime-mover and steering motors are respectively controlled in open- and closed-loop configurations.

\subsection{Design of the robot}

Educational robots are widely used in education of engineering fields, and numerous institutions employ robots to enhance learning efficiency and deepen the presented knowledge. Almost all of the robots constructed for this purpose in universities and companies make use of high-level and complicated electronics in order to implement the sub-functions. Usually, preprogrammed Micro-Controllers ( $\mu \mathrm{Cs}$ ) carry out the necessary processes, but the program can be modified by the students. In these robots, the education of electronics is provided by software and programming. Radio link is also done using advanced and relatively complex modules, which comprise several modulations and significant communication systems to enhance the quality of the link. Use of high-frequency carriers, usually within one of the Industrial, Scientific, and Medical radio (ISM) bands, makes the direct observation of signals using normal $20 \mathrm{MHz}$ oscilloscopes impossible.

Even by using an advanced and costly oscilloscope, the understanding of waves and signals would

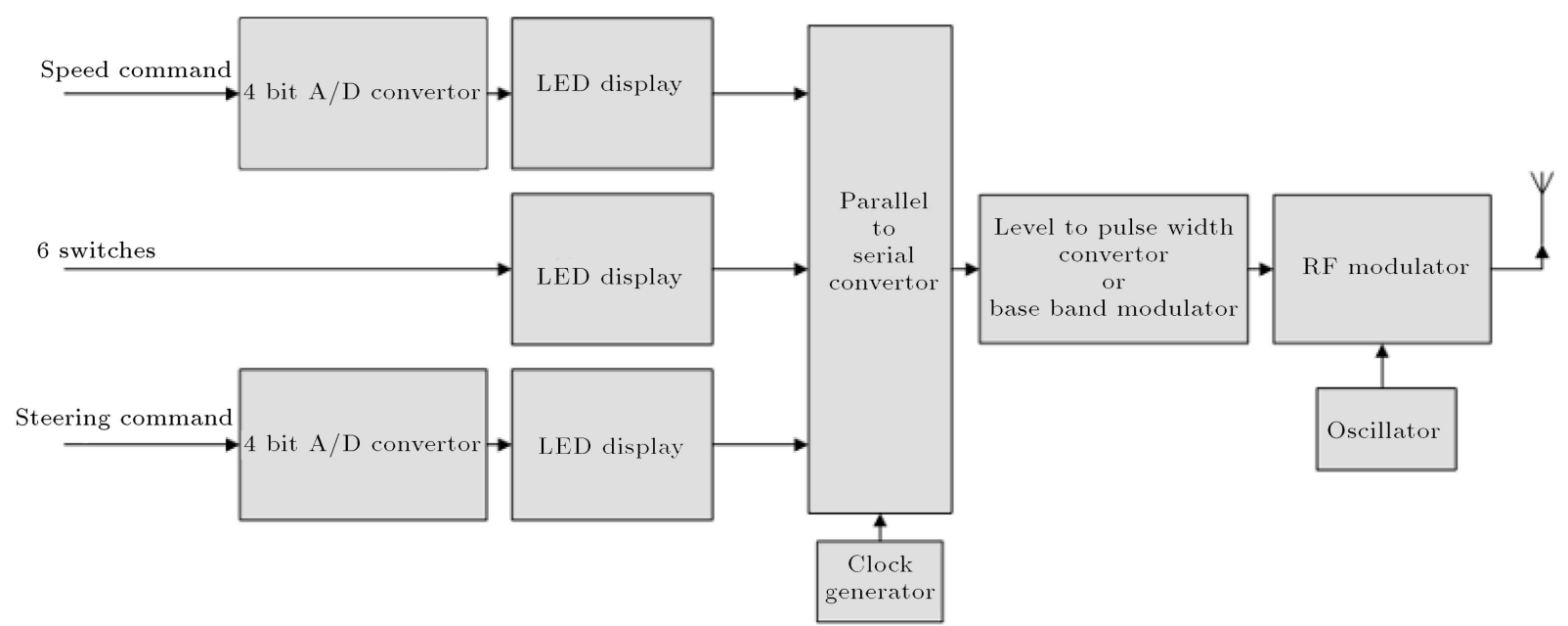

Figure 2. Block diagram of the control unit. 


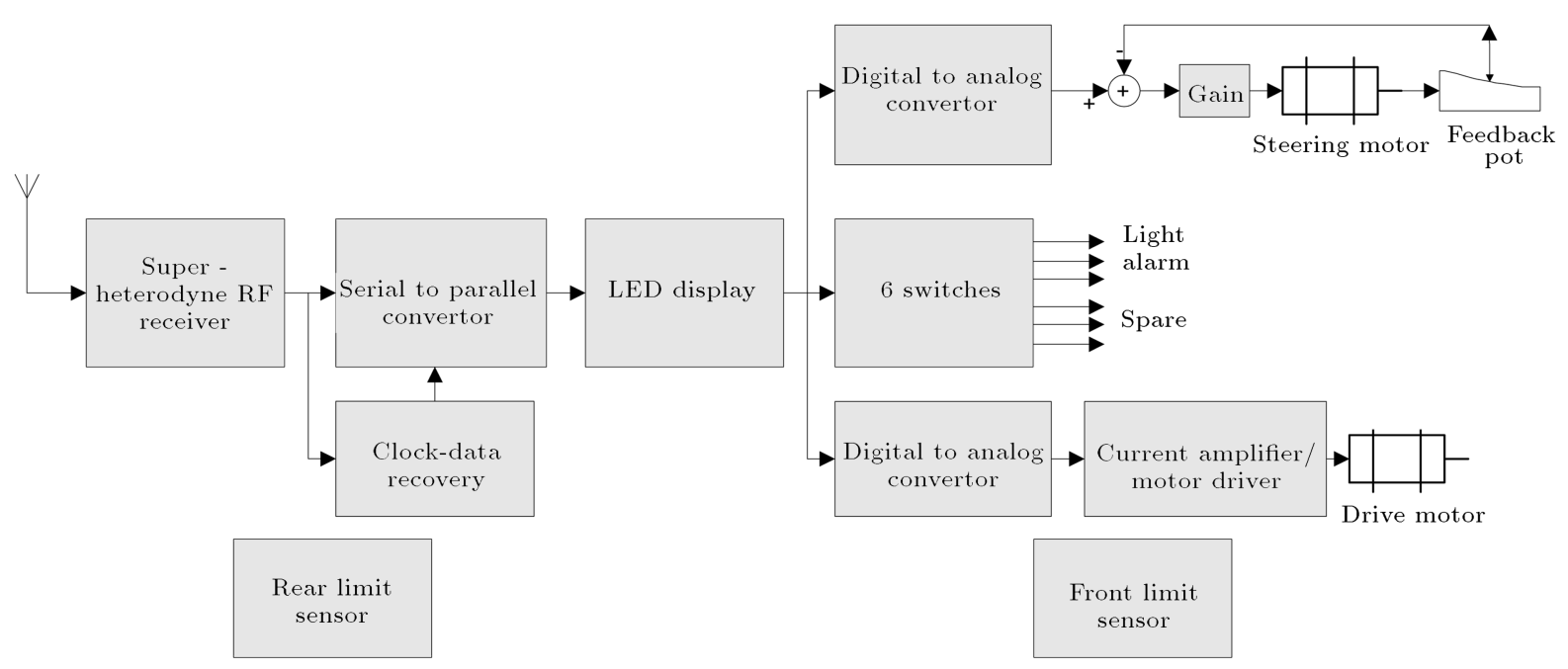

Figure 3. Structure of the robot car.

be far from reach of a freshman undergraduate student. Nevertheless, if a new student is not immediately and correctly exposed to the fundamental concepts, they would not obtain a deep and extendable knowledge of EE. Hence, the $27 \mathrm{MHz}$ carrier and amplitude modulation is selected.

The educational robot should have such an electronic design so that the students would be able to find a logical connection between the electronic circuits, observable signals, and introduced concepts in the class and, at the same time, carry out a perfect function in the real world. For these reasons, the designed robot has the following capabilities: motion in both (forward and backward) directions and rotation towards both (left and right) directions while having adjustable speed and steering angle. Furthermore, the robot displays some degree of intelligence, similar to a real robot, which prohibits falling or collision from the working desk, either from rear or front.

The robot employs simple electronics using either discrete elements or Low-Scale Integration (LSI) integrated circuits. The block diagrams of the robot as well as the control unit are printed on the seethrough package so that the student could identify the functionality and signal flow by inspection, and observe the waveforms of important signals using LEDs or a 2channel oscilloscope.

This educational robot illustrates examples of engineering disciplines ranging from human-machine interface, ergonomic, systematic and modular design, scalable design, and connection of Electrical to other engineering fields, such as Mechanical Engineering. Hence, the student is motivated to move towards a multi-disciplinary, rather than single-discipline, way of thinking in approaching engineering problems. This does not imply that all of the subjects are covered, but the focus has been to present useful examples from major engineering themes. In view of these attributes, Figure 4 illustrates the relation of each block in the system to various fields of EE.

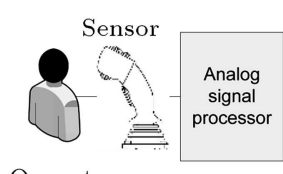

Operator

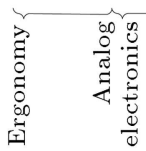

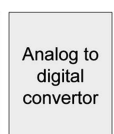

convertor
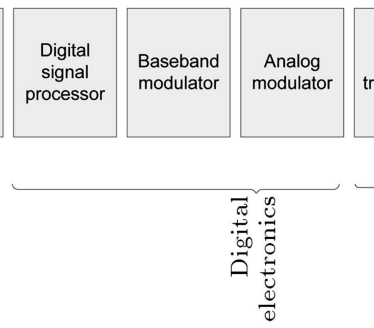
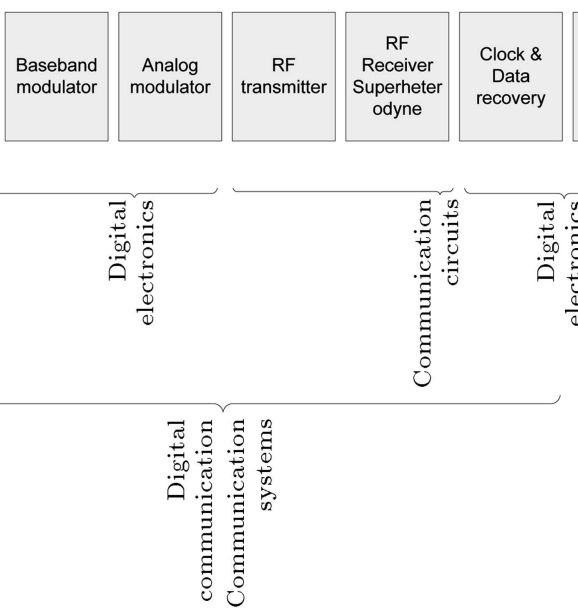
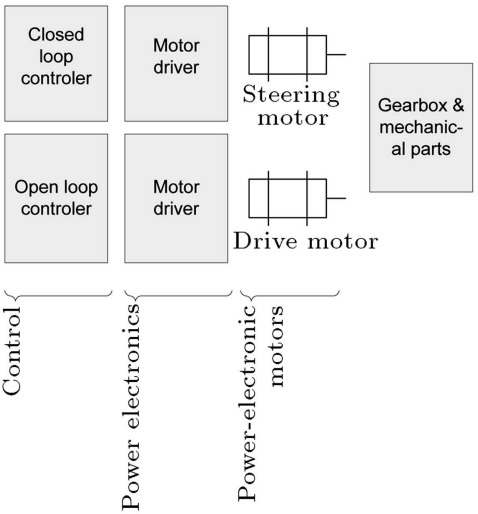

Figure 4. Relation of blocks and modules of the educational robotic system with various fields and subjects of EE. 
As the next step, addition of a bioelectric interface with the user to control the robot could provide an example of Bioelectrical Engineering. Similarly, addition of a solar or fuel-cell power source could also provide examples of renewable energy resources. Needless to say, several disciplines of Electrical Engineering, such as Energy Production, Transmission and Distribution Networks, Programmable Digital Processors, etc. remain, which are not covered.

\subsection{Usage}

The laboratory technician prepares the robot for the students. Test points, according to Figures 5 and 6 , are used to check out the signals and voltage levels.

\subsection{Experiments and observations on the robot}

In this section, a number of experiments and observations, which can be done on the aforementioned robot, are discussed. It is clear that this list does not include all of the possibilities. The robot has been designed so that the modules can be replaced, and based on their own capability and creativity, an interested student can modify the implementation of each module and observe the differences. All signals can be monitored and measured in real time as follows.

\subsubsection{Experiments on control unit}

1. Sensors: Two sensors to obtain the velocity and sensors commands from user, which are to be sent to the robot, are used. These sensors transform the angle to electrical voltage. Students measure the voltage and check the relation to input angle.

2. A/D converters: Output voltages from sensors are converted to digital signal using 4-bit A/D converters; the result is shown on LED bars. Students measure the input voltage and read the output number shown on LEDs and check the relation between them.

3. Parallel to serial conversion: Two command channels are already prepared from 4-bit A/D converters. There are 6 other bi-stable switches, 2 of which are used for the robot's beep and light and the rest are left free for students' use on purpose. Hence, there is a series of 14 bits of information in parallel. In order to transmit this data along a single communication link, the bits should be ordered into series. A3 test point shows the series signal. Students observe the serial data on oscilloscope and find specific data, e.g. steering command, in the serial stream.

4. Base band modulation: When the digital data is put in series, detection of similar and sequential bits can be difficult to comprehend. Difficulty could also mean that the receiver electronics may suffer from errors and this causes problems. Hence, another representation (based on bits) is chosen. In this method, each bit is coded as a pulse: Zero is represented by a pulse with smaller temporal duration or pulse width and one is represented by a pulse with larger temporal duration or pulse width.



Figure 5. Top view of the control unit. A1 through A9 are test points; A9 is considered for ground connection. In the bottom left, the phrase "data clock carrier" is printed over a tristable switch, which allows choosing between data and clock or a pure sinusoid for radio transmission. This feature makes the modulated signal or carrier readily available for viewing by a spectrum analyzer. 


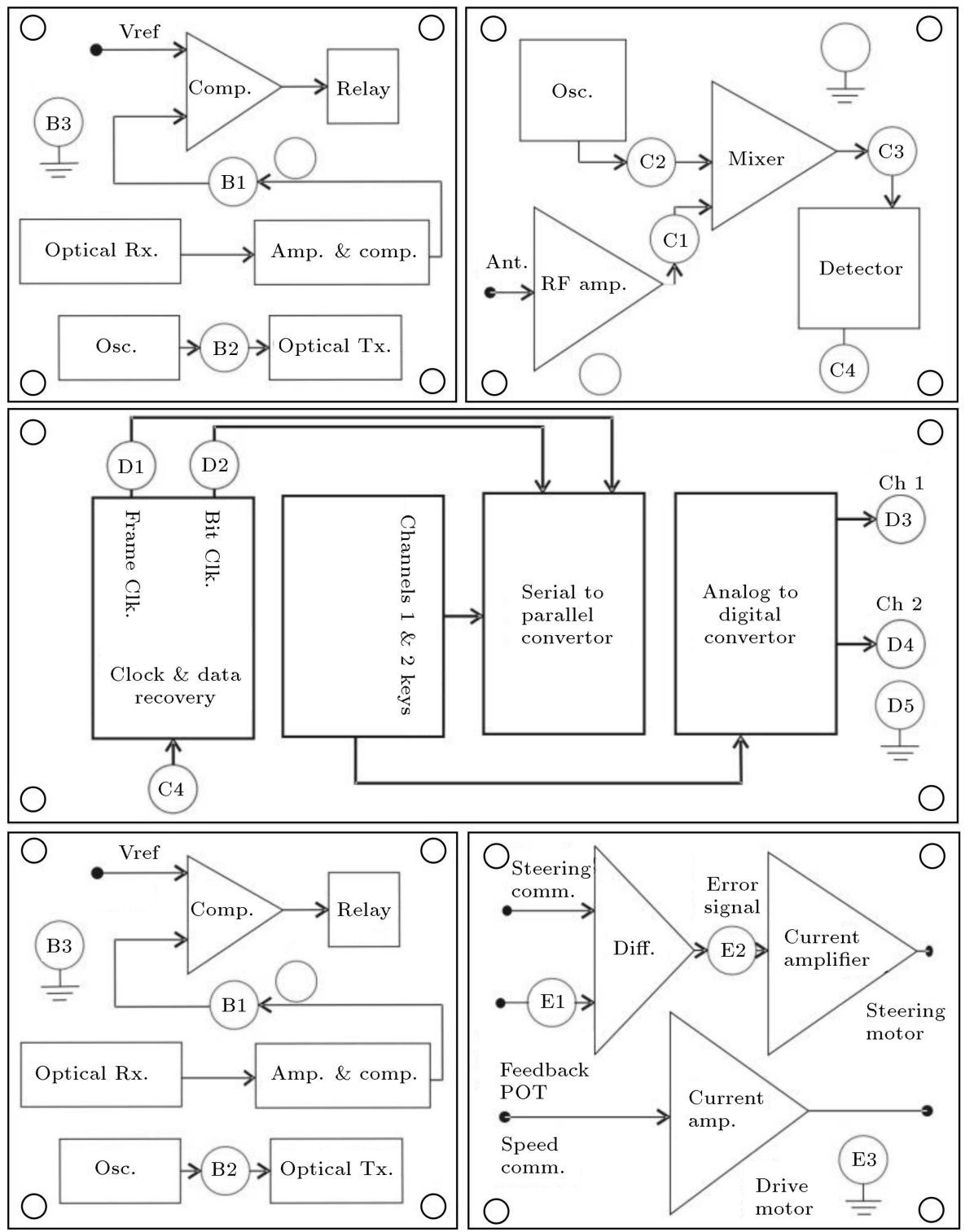

Figure 6. Top view of the electronic robot: Test points are shown similar to the control unit by characters and numbers.

This is the well-known PWM technique. Students observe both signals on oscilloscope and compare them to find out some benefits of this modulation.

5. Radio Communications: Now, information is ready for transmission to the robot. However, the information signal is too weak for this purpose. For propagation in the air, a carrier with a given fixed frequency is employed. Therefore, the signal $x(t)$ is multiplied by a sinusoid such as $\sin (\omega t)$. The amplitude-modulated signal $x(t) \sin (\omega t)$ is the simplest form of modulation, which can be easily seen on the oscilloscope. Using a special 3position switch, students can choose carrier, carrier modulated by a $1 \mathrm{kHz}$ clock, and actual signal. Lab Assistant will show students these signals on a spectrum analyzer. This helps students get insight into frequency domain concept and see the effect of amplitude modulation on signal in frequency domain.

\subsubsection{Experiments on robot}

6. Super-heterodyne receiver: In this educational robot, the transmitted signal is received and detected by a super-heterodyne receiver, as shown in Figure 7. In this method, the radio signal after amplification is multiplied by a sinusoid, which is in turn generated by a local oscillator. In this way, two sinusoids are obtained with sum and difference frequencies. This signal, after passage through a pass-band filter centered at the difference frequency (being set to $455 \mathrm{kHz}$ in the design), is reduced to the original amplitude-modulated signal $x(t) \sin (\omega t)$; however, the carrier frequency is now 


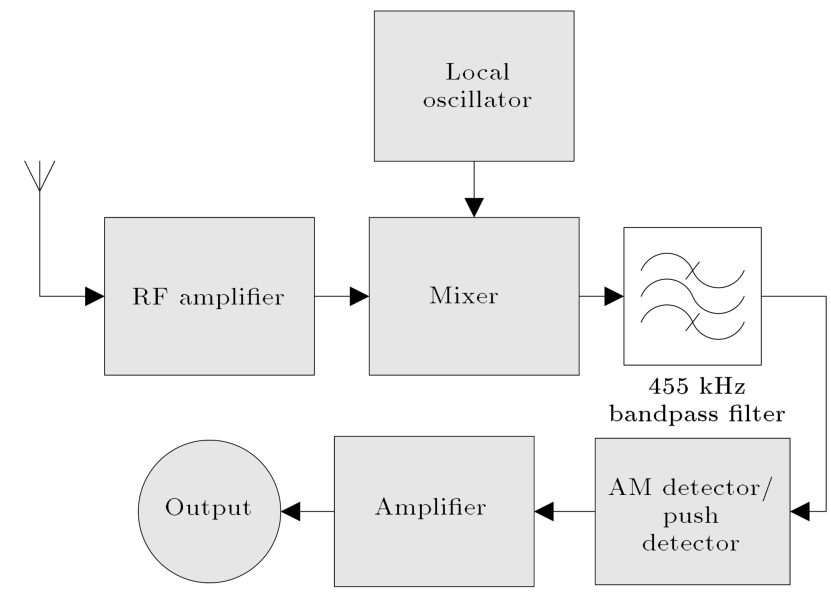

Figure 7. Super-heterodyne radio receiver: Output test points on the robot are marked as B1 through B4.

reduced from $27 \mathrm{MHz}$ to $455 \mathrm{kHz}$. This signal after detection constructs the base band signal. Students measure the signals using oscilloscope and interpret them.

7. Data Recovery in Receiver: Part $\mathrm{C}$ in the robot has the job to recover the serial data from transmitted signal. This information is shown on 14 LEDs, being exactly comparable to the LEDs on the control unit. LEDs corresponding to the velocity and direction channels are sent to $2 \mathrm{D} / \mathrm{A}$ converters to restore analogue control voltages. Students will see this signal and compare it with the original one fed to modulator.

8. Power section and motor drive: Usually, processing electronic circuits do not have enough currentdriving capability to feed power elements and devices such as motors. Hence, a motor driver is always needed to convert the control signal into a similar signal with the ability of high-current delivery. Students measure the signals and observe the relation of signal level to speed of tires.

9. Front steering angle control: In this experiment the student becomes familiar with an example of a closed-loop control system to fix the required quantity. In the previous experiment, if the angle of steering control was kept fixed, the moving speed of the robot would not be the same. For instance, depending on the roughness or inclination of the surface, speed may increase or decrease by applying the steering control. Here, a closed-loop system is employed to control applied steering signal. This is shown in Figure 8. In this system, a potentiometer is attached to the front wheels, and a voltage is developed in the potentiometer output proportional to the steering angle. This is subtracted from the control voltage. The result is the error signal, which is amplified and applied to the motor. In this case, motor always moves in the direction that causes reduction in error signal to zero. At the zero error signal level, the motor clearly stays at rest. Students observe the effect of feedback by comparing angle of wheels when the system is on or off. Also, they can explore effect of disturbance and loop gain using a potentiometer.

10. Intelligence: This robot is equipped with sensors for detection of desk borders and preventing the robot from crossing the limits. For this purpose, sensors are placed at the front and rear of the robot, which function in two similar circuits. These circuits cause a relay to activate and break the drive motor's circuit, while approaching a border (Figure 9). As shown here, when the robot reaches one of the borders, the corresponding switch is opened and the reverse diode stops the current flow and, therefore, motor rotation.

Finally, in Figure 10, a photo of the control unit is shown. Test points are also clearly visible on the top of each module.

\section{Experiments and observations}

The implemented system was in use at the School of EE at SUT for over 10 academic semesters, spanning more than 4 academic years. It was experimented by at least 6 groups each year, consisting roughly 200 freshman students of EE per annum, totaling over 1,000 individuals. Students had already become familiar with the concepts and they had experimented several discrete elements and simple electrical and electronic circuits.

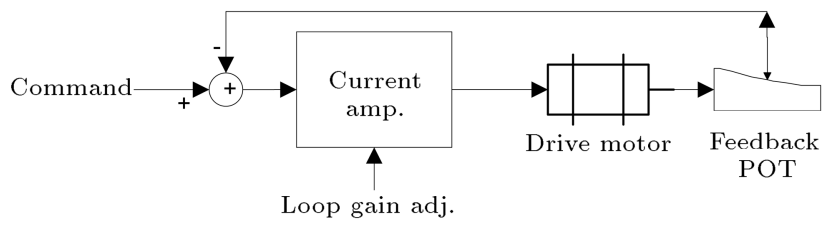

Figure 8. Closed-loop control system for front steering angle.



Figure 9. Control circuit for remaining within borders. 


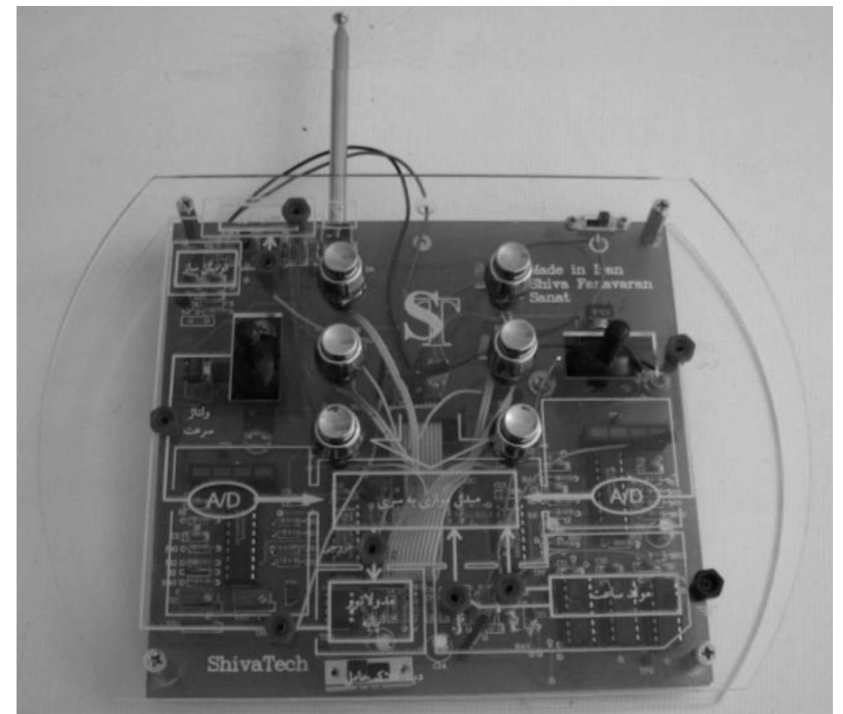

Figure 10. Photo of the control unit.

The experience of students after working with this system revealed that a stronger motivation in education and learning was being initiated. Evaluation forms and feedback from students showed that nearly all of the students and professors were satisfied by the design and robustness of the robot and control unit. It was found that experimenting with the robot had established a better connection between the preliminary knowledge they had acquired in the classroom and a real working electronic system through the bridge of creative thinking.

This general method of learning set forth in the course Principles of Electrical Engineering and Laboratory has therefore successfully replaced our old educational system, in which the students simply stored and memorized information without having practical ideas for applications, by a research-oriented and creativethinking based method. The educational robot has met all of the specific goals expected for the freshman students.

Similar education methodology has also been already applied and extended to other courses, including electronic circuits, analogue circuits, digital electronics, etc. Students who enroll in the new system have become interested in exploring far-reaching areas such as system design, human-machine interfacing, ergonomic, and economic aspects from the very beginning of their education. This proves that the education of new generations demands for novel learning methods, and it would be only through this approach that our graduates could contribute to the advancement in rather complicated problems in the future.

\section{Conclusions}

A robot was designed and implemented for use in the laboratory-based course Principles of Electrical Engineering, which helped the students to obtain a perspective of modular systems while demonstrating a clear relationship among various disciplines. Modules could be replaced, inputs/outputs and signals were traceable, and discrete electronic circuits were employed to maximize understanding.

\section{Acknowledgment}

The authors acknowledge the members of the Curriculum Revision Committee of the School of Electrical Engineering at Sharif University of Technology. Also, they are indebted to Reza Rahnavard and Davood Sadooghi at Shiva Tech for the assistance in implementation of robots. This work was supported in part by Sharif University of Technology and Iranian Ministry of Power.

\section{References}

1. Ryder, J.D. and Fink, D.G., Engineering and Electronics, IEEE, New York (1984).

2. Grinter, L.E. "Report on evaluation of engineering education (1952-1955)", J. Eng. Educ., 4(1), pp. 25-63 (1955).

3. Berry, F.C., DiPiazza, P.S., and Sauer, S.L. "The future of electrical and computer engineering education", IEEE Trans. Edu., 46(4), pp. 467-476 (2003).

4. Cavin, III, R.K., Joyner, Jr., W.H., and Wiggins, V.C. "A semiconductor industry perspective on future directions in ECE education", IEEE Trans. Edu., 46(4), pp. 463-466 (2003).

5. Hu, S.C. "A wholesome ECE education", IEEE Trans. Edu., 46(4), pp. 444-451 (2003).

6. Evans, D.L., Goodnick, S.M., and Roedel, R.J. "ECE curriculum in 2013 and beyond: vision for a metropolitan public research university", IEEE Trans. Edu., 46(4), pp. 420-428 (2003).

7. Goodnick, S.M. "Guest editorial: A vision for ECE education in 2013 and beyond", IEEE Trans. Edu., 46(4), p. 405 (Nov. 2003).

8. Somerville, M., Anderson, D., Berbeco, H., Bourne, J.R., Crisman, J., Dabby, D., Donis-Keller, H., Holt, S.S., Kerns, S., Kerns, Jr., D.V., Martello, R., Miller, R.K., Moody, M., Pratt, G., Pratt, J.C., Shea, C., Schiffman, S., Spence, S., Stein, L.A., Stolk, J.D., Storey, B.D., Tilley, B., Vandiver, B., and Zastavker, Y. "The olin curriculum: Thinking toward the future", IEEE Trans. Edu., 48(1), pp. 198-205 (2005).

9. Frolik, J. and Fortney, M. "A low-cost wireless platform for first-year interdisciplinary projects", IEEE Trans. Edu., 49(1), pp. 105-112 (2006). 
10. Hazzan, O., Tal, A., and Keidar, I. "Can a oneday conference change female high school students' perception of electrical engineering?", IEEE Trans. Edu., 49(3), pp. 415-416 (2006).

11. Huettel, L.G., Brown, A.S., Coonley, K.D., Gustafson, M.R., Kim, J., Ybarra, G.A., and Collins, L.M. "Fundamentals of ECE: A rigorous, integrated introduction to electrical and computer engineering", IEEE Trans. Edu., 50(3), pp. 174-181 (2007).

12. Hussmann, S. and Jensen, D. "Crazy car race contest: Multicourse design curricula in embedded system design", IEEE Trans. Edu., 50(1), pp. 61-67 (2007).

13. Plaza, I. and Medrano, C.T. "Continuous improvement in electronic engineering education", IEEE Trans. Edu., 50(3), pp. 259-265 (2007).

14. Kim, S.-H., Roh, C.-W., Kang, S.-C., and Park, M.Y. "A hybrid autonomous/teleoperated strategy for reliable mobile robot outdoor navigation", Proc. SICEICASE Int. Joint Conf., pp. 3120-3125, Busan (2006).

15. Blaer, P. and Allen, P.K. "TopBot: Automated network topology detection with a mobile robot", Proc. 2003 IEEE Int. Conf. Robotics \& Automation, pp. 1582-1587, Taipei (2003).

16. Shvartsman, A., Tedder, M., and Chung, C.-J. "A modular mobile robotic platform as an educational tool in computer science and engineering", Proc. Int. Conf. Comp., Commun., Control Technol. (CCCT), 5, pp. 314-317, Orlando (2003).

17. Golovinsky, A., Yim, M., Zhang, Y., Eldershaw, C., and Duff, D. "PolyBot and PolyKineticTM system : A modular robotic platform for education", Proc. 2004 IEEE Int. Conf. Robotics \& Automation, pp. 13811386, New Orleans (2004).

18. Maxwell, B.A. and Meeden, L.A. "Integrating robotics research with undergraduate education", IEEE Intel. Sys. Their Appl., 15(6), pp. 22-27 (2000).

19. Papadimitriou, V. and Papadopoulos, E. "Putting low-cost commercial robotics components to the testDevelopment of an educational mechatronics/robotics platform using LEGO components", IEEE Robotics \& Automation Magazine, 14(3), pp. 99-110 (2007).

20. Malec, J. "Some thoughts on robotics for education", 2001 A A A I Spring Symp. on Robotics and Edu., Stanford University (March 2001).

21. Padir, T. and Chernova, S. "Guest editorial special issue on robotics education", IEEE Trans. Edu., 56(1), pp. 1-2 (2013).

22. Aroca, R.V., Gomes, R.B., Tavares, D.M., Souza, A.A.S., Burlamaqui, A.M.F., Caurin, G.A.P., and Goncalves, L.M.G. "Increasing students' interest with low-cost CellBots", IEEE Trans. Edu., 56(1), pp. 3-8 (2013).

23. Gassert, R., Metzger, J.-C., Leuenberger, K., Popp, W.L., Tucker, M.R., Vigaru, B., Zimmermann, R., and Lambercy, O. "Physical student-robot interaction with the ETHZ haptic paddle", IEEE Trans. Edu., 56(1), pp. 9-17 (2013).
24. Kulich, M., Chudoba, J., Kosnar, K., Krajnik, T., Faigl, J., and Preucil, L. "SyRoTek-distance teaching of mobile robotics", IEEE Trans. Edu., 56(1), pp. 1823 (2013).

25. McLurkin, J., Rykowski, J., John, M., Kaseman, Q., and Lynch, A.J. "Using multi-robot systems for engineering education: Teaching and outreach with large numbers of an advanced, low-cost robot", IEEE Trans. Edu., 56(1), pp. 24-33 (2013).

26. Navarro, P.J., Fernandez, C., and Sanchez, P. "Industrial-like vehicle platforms for postgraduate laboratory courses on robotics", IEEE Trans. Edu., 56(1), pp. 34-41 (2013).

27. Vona, M. and Shekar, N.H. "Teaching robotics software with the open hardware mobile manipulator", IEEE Trans. Edu., 56(1), pp. $42-47$ (2013).

28. Cielniak, G., Bellotto, N., and Duckett, T. "Integrating mobile robotics and vision with undergraduate computer science", IEEE Trans. Edu., 56(1), pp. 48-53 (2013).

29. Correll, N., Wing, R., and Coleman, D. "A one-year introductory robotics curriculum for computer science upperclassmen", IEEE Trans. Edu., 56(1), pp. 54-60 (2013)

30. De Cristoforis, P., Pedre, S., Nitsche, M., Fischer, T., Pessacg, F., and Di Pietro, C. "A behavior-based approach for educational robotics activities", IEEE Trans. Edu., 56(1), pp. 61-66 (2013).

31. Riek, L.D. "Embodied computation: An activelearning approach to mobile robotics education", IEEE Trans. Edu., 56(1), pp. 67-72 (2013).

32. Cappelleri, D.J. and Vitoroulis, N. "The robotic decathlon: Project-based learning labs and curriculum design for an introductory robotics course", IEEE Trans. Edu., 56(1), pp. 73-81 (2013).

33. Crowder, R.M. and Zauner, K.-P. "A project-based biologically-inspired robotics module", IEEE Trans. Edu., 56(1), pp. 82-87 (2013).

34. Huang, H.-H., Su, J.-H., and Lee, C.-S. "A contestoriented project for learning intelligent mobile robots", IEEE Trans. Edu., 56(1), pp. 88-97 (2013).

35. Silva, E., Almeida, J., Martins, A., Baptista, J.P., and Neves, B.C. "Master's in autonomous systems: An overview of the robotics curriculum and outcomes at ISEP, Portugal", IEEE Trans. Edu., 56(1), pp. 98-102 (2013).

36. Shiller, Z. "A bottom-up approach to teaching robotics and mechatronics to mechanical engineers", IEEE Trans. Edu., 56(1), pp. 103-109 (2013).

37. Bonarini, A. and Romero, M. "Robotics and design: An interdisciplinary crash course", IEEE Trans. Edu., 56(1), pp. 110-115 (2013). 
38. Crenshaw, T.L.A. "Using robots and contract learning to teach cyber-physical systems to undergraduates", IEEE Trans. Edu., 56(1), pp. 116-120 (2013).

39. Hamblen, J.O. and van Bekkum, G.M.E. "An embedded systems laboratory to support rapid prototyping of robotics and the internet of things", IEEE Trans. Edu., 56(1), pp. 121-128 (2013).

40. Jung, S. "Experiences in developing an experimental robotics course program for undergraduate education", IEEE Trans. Edu., 56(1), pp. 129-136 (2013).

41. Yilmaz, M., Ozcelik, S., Yilmazer, N., and Nekovei, R. "Design-oriented enhanced robotics curriculum", IEEE Trans. Edu., 56(1), pp. 137-144 (2013).

42. Gil, A., Peidró, A., Reinoso, Ó., and Marín, J.M. "Implementation and assessment of a virtual laboratory of parallel robots developed for engineering students", IEEE Trans. Edu., 57(2), pp. 92-98 (2014).

43. Guo, Y., Zhang, S., Ritter, A., and Man, H. "A case study on a capsule robot in the gastrointestinal tract to teach robot programming and navigation", IEEE Trans. Edu., 57(2), pp. 112-121 (2014).

44. Gómez-de-Gabriel, J.M., Mandow, A., FernándezLozano, J., and García-Cerezo, A. "Mobile robot lab project to introduce engineering students to fault diagnosis in mechatronic systems", IEEE Trans. Edu., 58(3), pp. 187-193 (2015).

45. Dogmus, Z., Erdem, E., and Patoglu, V. "ReAct!: An interactive educational tool for AI planning for robotics", IEEE Trans. Edu., 58(1), pp. 15-24 (2015).

46. Scott, M.J., Counsell, S., Lauria, S., Swift, S., Tucker, A., Shepperd, M., and Ghinea, G. "Enhancing practice and achievement in introductory programming with a robot olympics", IEEE Trans. Edu., 58(4), pp. 249254 (2015).

47. Hassan, H., Domínguez, C., Martínez, J.-M., Perles, A., Capella, J.-V., and Albaladejo, J. "A Multidisciplinary PBL robot control project in automation and electronic engineering", IEEE Trans. Edu., 58(3), pp. 167-172 (2015).

48. Faria, C., Vale, C., Machado, T., Erlhagen, W., Rito, M., Monteiro, S., and Bicho, E. "Experiential learning of robotics fundamentals based on a case study of robot-assisted stereotactic neurosurgery", IEEE Trans. Edu., 59(2), pp. 119-128 (2016).

49. Aldebaran Robotics, https://www.ald.softbankrobotics.com/en

50. Ehsan, M., Tabandeh, M., Jahed, M., Haeri, M., Khalaj, B.H., Alavi, S.M.H., Faez, R., Fotuhi, M., Fardmanesh, M., Farzadeh, F., Nahvi, M., and Vakilian, M. "Curriculum revision committee at school of electrical engineering: Structural revision in electrical engineering education in the nation", J. Eng. Edu. Quarterly, 10(38), pp. 1-27 (2008) (in Persian).

\section{Biographies}

Mohsen Rahnavard received his BSc and MSc degrees in Electrical Engineering from Sharif University of Technology in 1994 and 1997, respectively, where he worked on ultrasonic transducers. He is the founder and Chief Executive Officer of Shiva Tech, with expertise in intelligent control systems and robotics.

Seyed Mohammad Hossein Alavi received the MSc degree from Karlsruhe University of Technology, Karlsruhe, Germany, in 1974, and the $\mathrm{PhD}$ degree in Electrical Engineering from Stuttgart University of Technology, Stuttgart, Germany, in 1993. Since 1993, he has been with the School of Electrical Engineering at Sharif University of Technology, Tehran, Iran, where he is currently an Emeritus Professor. He was a member of the technical staff at Siemens Instrumentations Laboratory, Wagner Computer Industry, WSWTrans, Fabrik, and Adler SMPS-Manufacturing, all in Germany, for several years. His research interests include switching power supplies and general electronic systems. Dr. Alavi was a recipient of the 1998 Distinguished Faculty Award.

Sina Khorasani received the MSc and PhD degrees in Electrical Engineering from Sharif University of Technology in 1996 and 2001, respectively, where he is now a full professor. He has been with the School of Electrical and Computer Engineering at Georgia Institute of Technology as a Postdoctoral (2002-2004) and Research Fellow (2011-2012). He is now with École Polytechnique Fédérale de Lausanne (2016-2017) as a visiting professor. His active research areas include quantum optics and photonics, and quantum electronics. Dr. Khorasani is a Senior Member of IEEE.

Mehdi Vakilian received the BSc degree in Electrical Engineering and the MSc degree in Electric Power Engineering from Sharif University of Technology, Tehran, Iran, in 1978 and 1986, respectively, and the $\mathrm{PhD}$ degree in Electric Power Engineering from Rensselaer Polytechnic Institute, Troy, NY, USA, in 1993. He was with the Iran Generation and Transmission Company, Tavanir, Iran, from 1981 to 1983, and then with Iranian Ministry of Energy from 1984 to 1985. Since 1986, he has been with the Department of Electrical Engineering at Sharif University of Technology, Tehran, Iran. During 2001 to 2003 and since 2014, he has been the chairman of the Department. From 2003 to 2004 and in a part of 2007 , he was on study leave at the School of Electrical Engineering and Telecommunications of the University of New South Wales, Sydney, Australia. He has been the director of the Power System Educational Group in the department for about 6 years. He has also been the director of a committee in charge of restructuring the Electrical Engineering Undergraduate Education at Sharif University of Technology, since 2007. His research interests are transient modeling of power system equipment, 
especially power transformers; optimum design of highvoltage equipment insulation; monitoring of power system equipment and their insulation; power system transients; and distribution system studies.

Mehdi Fardmanesh was born in Tehran, Iran, in 1961. He received the BSc degree from Tehran Polytechnic University, Tehran, Iran, in 1987, and the MSc and PhD degrees from Drexel University, Philadelphia, $\mathrm{PA}$, in 1991 and 1993, respectively, all in Electrical Engineering. In 1989, he joined Drexel University, where, until 1993, he conducted research in development of the thin- and thick-film high-temperature superconducting materials and devices, and development of ultralow-noise cryogenic characterization systems, where he was awarded a research fellowship by the Ben Franklin Superconductivity Center in 1989. From 1994 to 1996, he was Principal Manager for R\&D and the director of a private-sector research electrophysics laboratory, while also teaching in the Departments of Electrical Engineering and Physics at Sharif University of Technology, Tehran, Iran. In 1996, he joined the Electrical and Electronics Engineering Department, Bilkent University, Ankara, Turkey, teaching in the areas of solid-state and electronics, while also supervising his established Superconductivity Research Laboratory. In 1998 and 1999, he was invited to ISIForschungszentrum Juelich in Germany, where he pursued the development of low-noise high-Tc rf-SQUIDbased magnetic sensors. In 2000, he established an international collaboration between Bilkent University and Juelich Research Center in the field of superconductivity, and, from 2000 to 2004, he was the director of the joint project for development of high-resolution high-Tc SQUID-based magnetic imaging system. Since 2000, he has also reestablished his activities with the Electrical Engineering Department at Sharif University of Technology, where he is presently the head of the Electronics Department. He set up a Superconductor Electronics Research Laboratory (SERL) at Sharif University of Technology in 2003, which he still directs in the present time. His research interest has mainly focused on the design, fabrication, and modeling of high-temperature superconducting devices and circuits such as bolometers, microwave filters, and resonators; Josephson Junctions; and SQUID-based systems, in the areas of which he holds several international patents. 